\title{
Search for dark matter annihilation signals from dwarf spheroidal galaxies with H.E.S.S.
}

\author{
C. Farnier ${ }^{\star a}$, J.F. Glicenstein ${ }^{b}$, A. Jacholkowska ${ }^{c}$, E. Moulin ${ }^{b}$, and M. Vivier ${ }^{b}$ for the \\ H.E.S.S. Collaboration \\ ${ }^{a}$ Laboratoire de Physique Théorique et Astroparticules, CNRS/IN2P3, Université Montpellier II, \\ CC 70, Place Eugène Bataillon, F-34095 Montepllier Cedex 5, France \\ ${ }^{b}$ IRFU/DSM/CEA, CE Saclay, F-91191, Gif-sur-Yvette, Cedex, France \\ ${ }^{c}$ LPNHE, Université Pierre et Marie Curie Paris 6, Université Denis Diderot, Paris 7 , \\ CNRS/IN2P3, 4 Place Jussieu, F-75252, Paris Cedex 5, France \\ E-mail: farnierdin2p3.fr
}

\begin{abstract}
Dwarf spheroidal galaxies are among the most promising targets for indirect search of WIMP annihilation signal with very high energy (VHE) gamma-ray experiments. The absence of standard astrophysical sources in dwarf spheroidal galaxies considerably reduces the contamination from a possible exotic signal. The H.E.S.S. array of 4 Imaging Atmospheric Cherenkov Telescopes (IACT), situated in the Southern Hemisphere, has already observed some of these objects for energies above a few hundred GeV. After applying selection criteria, 18 hours of data are presently available for the analysis of Sagittarius dwarf galaxy. Results on the velocity-weighted annihilation cross-sections for Sagittarius dwarf are given in the framework of various Supersymmetric models (MSSM and mSUGRA) and for plausible dark matter halo profiles.
\end{abstract}

Identification of dark matter 2008

August 18-22, 2008

Stockholm, Sweden

\footnotetext{
* Speaker.
} 


\section{Introduction}

Cosmologists and astro-particle physicists consider that approximately $22 \%$ of the overall mass-energy budget of the Universe is composed of non-luminous and non-baryonic matter. The existence of this Dark Matter (DM) is supported by a large amount of observations such as rotation curves in spiral galaxies and velocity dispersion in elliptical galaxies, $\mathrm{X}$-ray emission, peculiar velocities of galaxies in galaxy clusters and weak lensing effects.

On galaxy scales, the proportion of this non-standard component can constitute $90 \%$ of the gravitational mass, whose microscopic nature remains one of the major mysteries in cosmology. This DM is usually assumed to be composed of stable, massive and weakly-interacting particles (WIMPs), with masses and annihilation cross-sections reproducing the right thermal abundances in the early Universe. Such candidates for WIMPs are proposed by models of particle physics [1].

Two cold dark matter (CDM) candidates, deriving from two different theories, are privileged: the lightest neutralino $\tilde{\chi}_{1}^{0}$ provided by supersymmetric extensions of the Standard Model with Rparity conservation [2] and the lightest Kaluza-Klein particle (LKP) [3] for extra dimension theories, most often the first KK mode of the hypercharge gauge boson, $\widetilde{B}^{(1)}$. These particles produce VHE $(\mathrm{E}>100 \mathrm{GeV}) \gamma$-rays in the final state of annihilation processes.

The High Energy Stereoscopic System (H.E.S.S.), an array of Imaging Atmospheric Cherenkov Telescopes (IACT), designed for high sensitivity in the $100 \mathrm{GeV}-10 \mathrm{TeV}$ energy range, is a suitable detector for VHE $\gamma$-rays and investigation of their possible origin.

\section{Dark Matter targets}

The IACT experiments have a field of view limited to a few degrees. In this context, all sky observations are not conceivable and only potential targets with high concentration of DM must be considered in priority. Different sources fill this requirement: the Galactic Center (GC) for which the DM component contribution to the $\gamma$-rays signal has already been constrained by H.E.S.S. [ 7 ]. Galaxy clusters are also good candidates due to the very large amount of DM expected. The Virgo cluster, M87, observed by H.E.S.S. shows a $\gamma$-ray emission in the VHE range. The observed flux is considerably higher than the DM predictions and spectrum characteristics exclude DM as the main component [5]. As those objects may contain astrophysical sources already known as VHE $\gamma$-ray emitters with flux generally stronger than expected from DM, it is hard to put any constraint on the nature of DM from these data.

On the contrary, dwarf spheroidal (dSph) galaxies present a high concentration of DM [6], and negligible standard $\gamma$-ray background, due to very poor amount of stellar gas and little dust. As the observed flux is proportional to the distance from the source, it must be highlighted that 4 already known dSph are distant by less than $100 \mathrm{kpc}$ from the GC.

The H.E.S.S. experiment has presently observed three different dwarfs, Sagittarius (Sgr) dSph for 18h cumulated in 2006 and 2007, Canis Major (CMa) (which denomination as a dSph is still controversial) for $\sim 10 \mathrm{~h}$ in 2007 and Carina $\mathrm{dSph}$ for $\sim 3 \mathrm{~h}$ at the beginning of 2008.

Since Carina observations are actually very limited and Canis Major suffers great uncertainties, both on its DM mass content and on its DM density profile, we will concentrate here mostly on Sagittarius dSph. 


\section{H.E.S.S. observations of Sagittarius dwarf spheroidal galaxy (Sgr dSph)}

The H.E.S.S. array in Southern hemisphere is composed of four Imaging Cherenkov Telescopes which allows the use of the stereoscopic technique. Associated to the great collection surface $\left(107 \mathrm{~m}^{2}\right)$ of each telescope and fine pixellisation of cameras (960 PMTs per camera), it confers to H.E.S.S. unequaled performance in the VHE range of $\gamma$ astronomy. The total field of view of H.E.S.S. is $\sim 5^{\circ}$, allowing so far the study of extended sources. The stereoscopic technique improves the reconstruction of the incoming direction of the primary gamma and its incident energy. It leads also to an excellent rejection of the hadronic background produced by charged cosmic rays.

The $\gamma$-ray data obtained with H.E.S.S. of the Sgr dSph galaxy consist of 18 hours of excellent quality observations, performed in June 2006 and May 2007, which have passed the selection criteria described in [7]. The average zenith angle for those observations is $16^{\circ}$, which corresponds to an energy threshold of $\sim 250 \mathrm{GeV}$ for the performed analysis.

Gamma-ray and hadronic showers were reconstructed from their images in Cherenkov light, using two different methods. The first one is based on the Hillas parameters [8] characterizing each image globally, the second one on a pixel-per-pixel comparison of the image with a template generated by a semi-analytical model of electromagnetic showers [9]. For this analysis, the separation between gamma candidates and hadrons is done using a combination of the Hillas parameters and the so-called Model goodness-of-fit, obtained via a minimisation of a likelihood function. The typical order of magnitude for the energy resolution obtained with both analyses is $\sim 15 \%$. An additional cut on the primary interaction depth is also used to improve background rejection.

In both cases, no signal was found neither at the nominal position of Sgr dSph, which was chosen at the position of Sgr luminous cusp coincident with the globular cluster M54, nor in the whole field of view of the camera confirmed by a flat $\theta^{2}$ distribution of the observed $\gamma$-ray events relative to the target position.

This results lead to an upper limit in the number of $\gamma$-rays that can have been emitted by Sgr dSph. Using the Feldman and Cousins procedure [10], we obtain a $95 \%$ confidence level (CL) upper limit:

$$
N_{\gamma}^{95 \% C . L .}=82
$$

\section{4. $\gamma$-ray predictions for CDM annihilation and model exclusion limits}

The differential flux of $\gamma$-rays from the WIMPs annihilation can be expressed as:

$$
\frac{d \Phi}{d E}(\Delta \Omega, E)=\frac{1}{4 \pi} \frac{<\sigma v>}{m_{D M^{2}}} \frac{d N_{\gamma}}{d E_{\gamma}} \times \overline{\mathrm{J}}(\Delta \Omega) \Delta \Omega
$$

The first part corresponds to the particle physic model, the second one to the astrophysical factor which is related to the DM density profile of the source.

In the astrophysical factor, $\mathrm{J}$ is the integral along the line of sight (l.o.s) of the squared density of the DM distribution in the object. $\overline{\mathrm{J}}$ is its average over the solid angle of the integrated region, which is $\Delta \Omega=2 \times 10^{-5} \mathrm{sr}$ for H.E.S.S. since we are looking for a pointlike signal:

$$
\overline{\mathrm{J}}(\Delta \Omega)=\frac{1}{\Delta \Omega} \int_{\Delta \Omega} \mathrm{d} \Omega \int_{\text {l.o.s }} \mathrm{ds} \rho^{2}(\mathrm{~s})
$$


Two different modeling of the dark matter halo of Sgr dSph have been considered in order to cover a large range of $\overline{\mathbf{J}}$ due to important astrophysical uncertainties on the halo density profile of Sgr dSph. The first one is a cuspy Navarro Frenck White (NFW) parametrization, [11], for which conservative parameter values were taken from [12]. Another type of halo considered to describe Sgr dSph is a core profile discussed in [12] with parameter values determined by using data of [13] (the core radius $r_{c}$ is only an upper limit). Assuming a velocity dispersion independent from the position and taking the value of the central velocity dispersion of Sgr dSph given in [14], a set of parameters is reported in the table 1. As the determined region of this core profile is very small, the value of $\overline{\mathbf{J}}$, calculated via the equation 4.2 , is quite large, as shown in table 1 (more details of this modeling can be found in [15]).

\begin{tabular}{|c|c|c|c|c|}
\hline Halo type & Density function & Parameters & $\begin{array}{c}\overline{\mathbf{J}} \\
\left(10^{24} \mathrm{GeV}^{2} \mathrm{~cm}^{-5}\right)\end{array}$ & $\begin{array}{c}\text { Fraction of signal } \\
\text { in } \Delta \Omega=2 \times 10^{-5} \mathrm{sr}\end{array}$ \\
\hline \hline Cuspy NFW & $\rho_{N F W}(r)=\frac{A}{r\left(r+r_{s}\right)^{2}}$ & $\begin{array}{c}r_{s}=0.2 \mathrm{kpc} \\
\mathrm{A}=3.3 \times 10^{7} M_{\odot}\end{array}$ & 2.2 & $93.6 \%$ \\
\hline Cored & $\rho_{\text {core }}(r)=\frac{v_{a}^{2}}{4 \pi G} \frac{3 r_{c}^{2}+r^{2}}{\left(r_{c}^{2}+r^{2}\right)^{2}}$ & $\begin{array}{c}r_{c}=1.5 \mathrm{pc} \\
v_{a}=13.4 \mathrm{~km} \mathrm{~s}^{-1}\end{array}$ & 75.0 & $99.9 \%$ \\
\hline
\end{tabular}

Table 1: Structural parameters for a cuspy NFW $\left(r_{s}, \mathrm{~A}\right)$ and a cored $\left(r_{c}, v_{a}\right)$ DM halo model, respectively. The values of the solid-angle-averaged l.o.s integrated squared DM distribution are reported in both cases for the solid angle region $\Delta \Omega=2 \times 10^{-5} \mathrm{sr}$.

Using the value of $N_{\gamma}^{95 \% C . L .}$ and equation 4.1, we can derive constraints on the velocityweighted cross-section of DM annihilation signal:

$$
<\sigma v>_{\min }^{95 \% C L}=\frac{4 \pi}{T_{o b s}} \frac{m_{D M}^{2}}{\overline{\mathrm{J}}(\Delta \Omega) \Delta \Omega} \frac{N_{\gamma}^{95 \% C L}}{\int_{0}^{m_{D M}} A_{e f f}\left(E_{\gamma}\right) \frac{d N}{d E_{\gamma}} d E_{\gamma}}
$$

where $T_{o b s}$ is the observation time of the source and $A_{\text {eff }}$ is H.E.S.S. effective area.

Depending on the considered distribution of the CDM in the Sgr dSph halo, the constraints have been calculated and compared to the predictions for phenomenological MSSM (pMSSM) models as well as for models in which the supersymmetry breaking is mediated through gravitational interaction, mSUGRA. In both cases, models have been provided by the DarkSUSY4.1 program [16], interfaced with ISAJET 7.69 [17] in case of mSUGRA. The results are shown in figure 1. The few hours of observations available cannot constrain any mSUGRA model for the two halo density profiles discussed previously. This is also the case for pMSSM models considering the NFW cuspy profile. However, the core profile, already allows to exclude models with relic density values below WMAP constraints (red line).

\section{Conclusion}

The H.E.S.S. experiment has observed three dwarf spheroidal galaxies, which are among the best targets for DM detection as a very low standard background contamination is expected. The most constraining observations are related to $\mathrm{Sgr}$ dSph galaxy. The H.E.S.S. results reveal no 

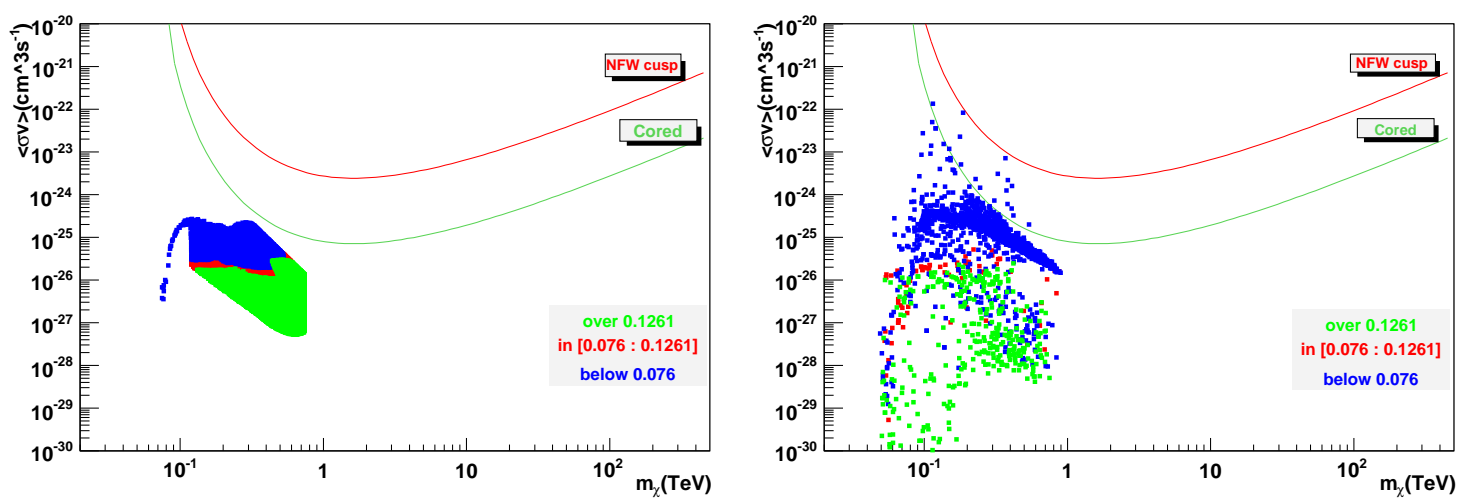

Figure 1: Upper limits at 95\% C.L. on $\langle\sigma v\rangle$ versus the DM particle mass in the case of a cusped NFW (red line) and a cored (green line) DM halo profiles respectively. The space parameter above the line are excluded by these observations. Left: mSUGRA parameter space explored with DarkSUSY 4.1 [16] interfaced with ISAJET 7.69 [17]. Right: pMSSM parameter space was explored with DarkSUSY 4.1 [16]. Each point on the plots corresponds to a specific model. Models satisfying WMAP relic density constraints, under and above are respectively displayed in red, blue and green. The parametrisation used to describe the spectrum in order to derive the constraints is taken from [18].

significant $\gamma$-rays excess at the nominal target position. However, assuming a quite optimistic core profile, some pMSSM models, with low relic density can already be excluded. The resulting $\langle\sigma v\rangle$ constraints from the $9.6 \mathrm{~h}$ of observations performed by H.E.S.S. in 2007 on CMa dwarf galaxy also do not allow to conclude on any SUSY or Kaluza-Klein model [19]. The next phase of the H.E.S.S. experiment including a fifth telescope of $\oslash 28 \mathrm{~m}$ will allow to gain in sensitivity and lower the threshold energy to $50 \mathrm{GeV}$, thus leading to better constraints on WMAP compatible models.

\section{Acknowledgements}

The support of the Namibian authorities and of the University of Namibia in facilitating the construction and operation of H.E.S.S. is gratefully acknowledged, as is the support by the German Ministry for Education and Research (BMBF), the Max Planck Society, the French Ministry for Research, the CNRS-IN2P3 and the Astroparticle Interdisciplinary Programme of the CNRS, the U.K. Science and Technology Facilities Council (STFC), the IPNP of the Charles University, the Polish Ministry of Science and Higher Education, the South African Department of Science and Technology and National Research Foundation, and by the University of Namibia. We appreciate the excellent work of the technical support staff in Berlin, Durham, Hamburg, Heidelberg, Palaiseau, Paris, Saclay, and in Namibia in the construction and operation of the equipment.

\section{References}

[1] G. Bertone, D. Hooper, J. Silk, 2005, Phys. Rep., 405, 279

[2] G. Jungman, K. Kamionkowski, K. Griset, 1996, Phys. Rep. 276, 195

[3] G. Servant \& T. Tait, 2003, Nucl. Phys. B, 605, 391 
[4] F. A. Aharonian et al. (H.E.S.S. Collaboration), 2006, Phys. Rev. Lett., 97, 221102

[5] F. A. Aharonian et al. (H.E.S.S. Collaboration), 2006, Science, 314, 1424

[6] M.I. Wilkinson et al., 2005, Proc. of XXI ${ }^{s t}$ IAP Colloqium: Mass Profiles \& Shapes of Cosmological Structures, Paris

[7] F. A. Aharonian et al. (H.E.S.S. Collaboration), 2006, Astron. \& Astrophys., 457, 899

[8] F. A. Aharonian et al. (H.E.S.S. Collaboration), 2005, Astron. \& Astrophys., 430, 865

[9] M. de Naurois et al., 2003, In International Cosmic Ray Conference

[10] G. Feldman \& R. Cousins, 1998, Phys. Rev. D., 57:3873

[11] J. Navarro et al., 1997, Astroparticle Journal, 490:493

[12] N. W. Evans et al., 2004, Phys. Rev. D., 69:123501

[13] L. Monaco et al., MNRAS, 356:1396

[14] S. Zaggia et al., 2004, Mem. Soc. Astr. It. Suppl., 5:291

[15] F. A. Aharonian et al. (H.E.S.S. Collaboration), 2008, Astropart. Phys. 29, 55-62

[16] P. Gondolo et al., 2004, JCAP, 0407:008

[17] H. Baer, F. E. Paige, S. D. Protopescu, X. Tata, arXiv:hep-ph/0312045

[18] L. Bergstrom, P. Ullio, J. Buckley, 1997., Astropart.Phys.9:137-162,1998. (astro-ph/9712318)

[19] F. A. Aharonian et al. (H.E.S.S. Collaboration), 2009, Astrophys. J., 0809.3894 